\title{
Microscopic Simulation of the Cardiac Electrophysiology: A Study of the Influence of Different Gap Junctions Models
}

\author{
Pierre-Elliott Bécue ${ }^{1,2,3}$, Mark Potse ${ }^{1,2,3}$, Yves Coudière ${ }^{1,2,3}$ \\ ${ }^{1}$ INRIA Bordeaux Sud-Ouest, Talence, France \\ ${ }^{2}$ Institut de Mathématiques de Bordeaux, Talence, France \\ ${ }^{3}$ Institut de Rythmologie et Modélisation Cardiaque, Pessac, France
}

\begin{abstract}
It has been suggested in the literature that a disorganisation of cardiac tissue at the cellular scale may affect the propagation of the action potential (AP) at the tissue scale, and may play a role in arrhythmia.

We developed a model of the myocardium at sub-cellular resolution in which the intracellular space, the cell membrane, and the extracellular space are discretized individually [1]. We present in this article an improvement of this model, including gap junction models at any interface between cells. We then test this approach on hand-crafted two-dimensional networks of a hundred of cells, and compare it with our previous model which did not include gap junctions modelling.
\end{abstract}

\section{Introduction}

The standard bidomain or monodomain equations model a cardiac tissue at the macroscopic scale. They may be derived by homogenization of the microscopic bidomain equations, which model the propagation of the cardiac action potential (AP) on a network of individual cells. For homogenization purposes, the network is assumed to be periodic.

Yet, dysfunction or disorganization (non periodicity) of the tissue at the cellular level may affect the propagation of the AP at the tissue scale. For instance, zigzag propagation at a cellular level has been hypothesized to lead to arrhythmias [2]. These alterations cannot be represented by the homogenized bidomain or monodomain models.

To understand and tackle such questions, we planned to simulate the bidomain equations written at the cellular scale on a manually designed network of cells. The equations were first studied theoretically [3], we proved existence of a solution [4], then we ran simulations on small handcrafted networks of individual cells [1].

In this paper we present an improved version of the equations presented in [1], that includes a proper gap junc- tions model. We briefly describe its properties, and then compare the results of both models.

\section{The bidomain model at the cell scale}

We consider a set of cells indexed from 1 to $N$. Each cell is a connected medium $\Omega_{i}$. The extracellular medium is denoted by $\Omega_{0}$, and assumed to be connected. Each cell $\Omega_{i}$ may be glued to some others. In such a case, their common interface is a gap junction, denoted by $\Gamma_{i j}$ (which is then the same as $\Gamma_{j i}$ ) where $j$ is the index of the other cell. The interface between a cell and the extracellular medium is the cell membrane denoted by $\Gamma_{0 i}$. An example is given Figure 1.

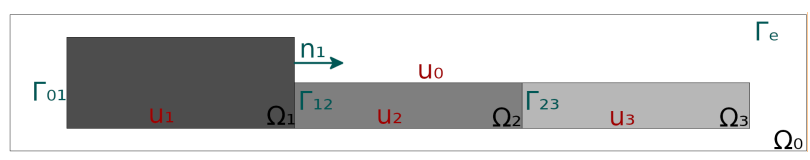

Figure 1. A 3 glued cells example describing the problem. The first cell is bigger because in our simulations, we want to be able to apply a stimulation on this cell without impacting the others.

For all time $t>0$, the microscopic equations are $-\nabla$. $\left(\sigma_{i} \nabla u_{i}\right)=0$ for all $i \in\{0, \ldots, N\}$. The transmission conditions between two domains $\Omega_{i}$ and $\Omega_{j}$ is

$$
-\sigma_{i} \nabla u_{i} \cdot n_{i}=\sigma_{j} \nabla u_{j} \cdot n_{j}=c_{i j} \partial_{t} v_{i j}+F_{i j}\left(v_{i j}, w_{i j}\right) .
$$

Last, there is a no-flux boundary condition $\sigma_{\mathrm{e}} \nabla u_{\mathrm{e}} \cdot n_{\mathrm{e}}=0$ on the external boundary $\Gamma_{\mathrm{e}}$ of the system. Here, $u_{i}$ denotes the potential field in the cell $i$ (or the extracellular medium 0). $\sigma_{i}$ are scalar electrical conductivities for each medium. The trans-membrane voltage across $\Gamma_{i j}$ is $v_{i j}=u_{i}-u_{j}$. Any interface $\Gamma_{i j}$ is modeled as a capacitance $c_{i j}$ in parallel with an ionic current $F_{i j}$. The vector $w_{i j}$ defined on the interface gathers the additional state variables of such an ionic current model, which is either a classic ionic model when $i$ or $j$ is 0 , or a gap 
junction model otherwise. We assume that, the initial data are given only for the trans-membrane voltages $v_{i j}$, as $v_{i j}(0, x)=v_{i j}^{0}(x)$ for $x \in \Gamma_{i j}$.

There exists a well posed weak formulation of these equations, where $u_{i}$ is searched in the space $H^{1}\left(\Omega_{i}\right)$, with an additional gauge condition for the sake of uniqueness.

We approximate the solution with the P1-Lagrange finite element method, and use an Euler time-stepping method, implicit on the diffusion terms, and explicit on the ionic ones. Hence, for a sequence of times $t^{n}=n \delta t$ $(\delta t>0)$, we solve the discretized equations on each $u_{i}$ in the discrete spaces P1, with the condition that the meshes of each of the domains (cells, and the extracellular medium) match one with another.

For each time step, we have to solve a linear system of equations of the form $A U^{n}=F^{n}$ where $U^{n}=$ $\left(U_{0}^{n}, \ldots, U_{N}^{n}\right)^{T}$ is the vector of the degrees of freedom, and the right-hand side vector $F^{n}=\left(F_{0}^{n}, \ldots, F_{N}^{n}\right)^{T}$ involves the nonlinear functions $F_{i j}$.

\section{Simulations and results}

We intend to compare our results with the ones we got from our previous work in [1]. Hence, we build two kind of problems. One type where the cells are geometrically connected by channels of intracellular material (see Figure 2), and one is with "glued" cells (as in fig. 1, or more generally fig. 3). In the former model, there is only one long cell with local reductions that model GJ channels. This is not a realistic model of gap junctions since the channels are too wide. In both figures, the "S" shape of the cell network is here for readability and also to respect the constraint that the extracellular medium has to be connected. On the latter

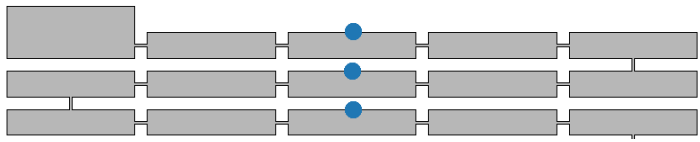

Figure 2. The top subset of a 100 cells network with geometrical GJCs. The blue dots are spots where we measure the transmembrane voltage along time. The whole network expands on 20 lines.

model, each node on an interface between cells is assumed to be a gap junction. We will first test a linear gap junction model (Linear GJ), ie $F_{i j}\left(v_{i j}, w_{i j}\right)=v_{i j} / R_{i j}$, where coefficients $R_{i j}$ are the conductances of the gap junctions. Then, we will show results with a non-linear gap junction model designed after experimentations on mouse cells [5]. In the simulations, the intracellular conductivities $\sigma_{i}$ are set to $1.7 \mathrm{mS} \mathrm{cm}^{-1}$ and the extracellular conductivity $\sigma_{e}$ is set to $3.0 \mathrm{mS} \mathrm{cm}^{-1}$. The membrane capacitance $C_{m}$ is

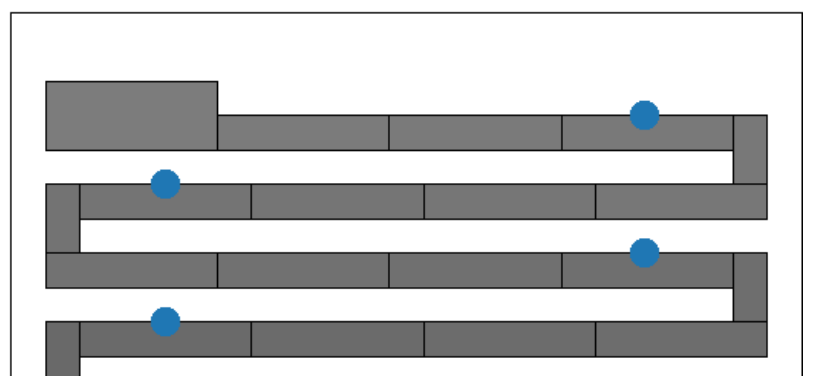

Figure 3. The top subset of a 99 glued cells network. The blue dots are spots where we measure the transmembrane voltage along time. The darkness of the cells is proportionate to their index (from 1 to 99 ). The whole network expands on 20 lines, the last lacking one cell compared to the others.

set to $1 \mu \mathrm{F} \mathrm{cm}{ }^{-2}$.

\subsection{Specificities of the linear system}

The symmetric matrix of the linear system is a combination of a stiffness matrix computed on all nodes and a mass matrix, computed on the nodes on any interface $\Gamma_{i j}$. Due to the coupling conditions between the cells, this mass matrix has specific terms. It is challenging to compute these matrices efficiently in our finite-element code, CEPS.

In addition, the initial problem is a pure Neumann equation (no-flux condition on the external boundary). Hence the linear system has a unique solution defined up to a constant. Here we compute the solution perpendicular to the kernel space of the matrix $A$ (corresponding to a gauge condition) with a conjugate gradient iterative solver, as suggested in ref [6].

\subsection{Computational solver and meshes}

Our model was implemented in the software code CEPS developed at Inria. It relies on the PETSc library to solve the linear systems on parallel computers. In test cases below, the problems were designed via a Python script, and meshed using the Triangle meshing software. For our simulations, the channel-version of our meshes had $34 \mathrm{k}$ nodes, $67.6 \mathrm{k}$ triangles. The glued one had $32 \mathrm{k}$ nodes and $63.4 \mathrm{k}$ triangles.

\subsection{Comparison between the channel and the Linear GJ cases}

We chose a resistance value $R_{i j}$ for the linear GJ case iteratively. Indeed, the bibliographic references always provide a resistance per unit area. Here, in two-dimensions, we do not have any precise value for the area. Furthermore, we don't really know the density of gap junctions on 
the interfaces. We hence chose a value that gave reasonable action potential propagation velocities, $R_{i j}=0.015 \mathrm{k} \Omega$. For each case, we run a simulation for $420 \mathrm{~ms}$, with a time step of $0.05 \mathrm{~ms}$, and an output each $0.1 \mathrm{~ms}$. In each case, we apply a stimulation as a current between each side of the upper-left cell. This stimulation occurs at $t=20 \mathrm{~ms}$. For the Linear GJ case, the intensity of the stimulation is $2.25 \times 10^{-5} \mu \mathrm{A}$ during $0.15 \mathrm{~ms}$. For the channel case, the stimulation intensity is $2.70 \times 10^{-5} \mu \mathrm{A}$ for the same duration. This difference results from the fact that in the channel case, the diffusion implies the need for a bigger stimulation. We show in Figures 4 and 5 the depolarization of the cells, measured on the blue dots shown in Figures 2 and 3 respectively.

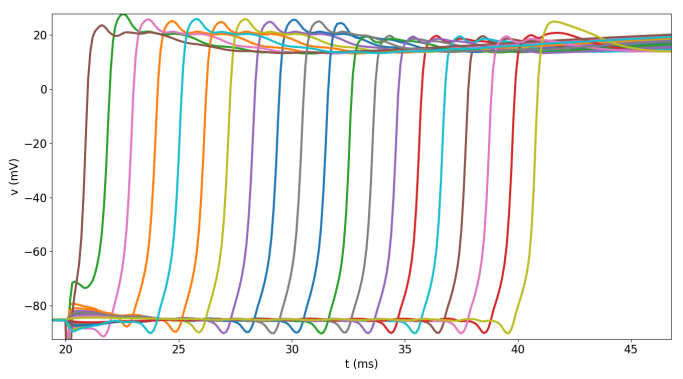

Figure 4. Voltages measured on the blue dots shown in Figure 2 for the channel problem. The action potential takes $20 \mathrm{~ms}$ to cross $0.855 \mathrm{~cm}$, which gives a velocity of $42.72 \mathrm{~cm} \mathrm{~s}^{-1}$, compatible with the literature.

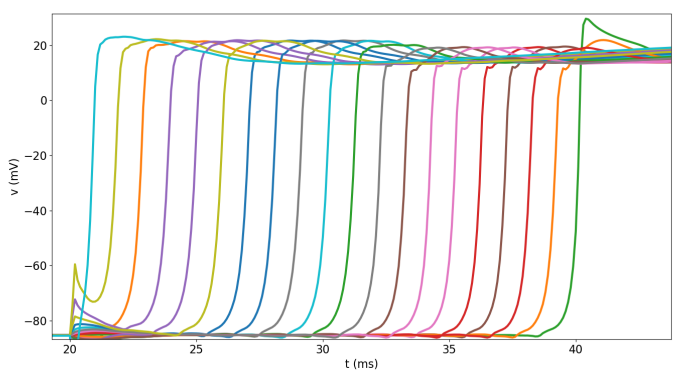

Figure 5. Voltages measured on the blue dots shown in Figure 3 for the linear GJ problem. The action potential takes $19 \mathrm{~ms}$ to cross $0.817 \mathrm{~cm}$, which gives a velocity of $43 \mathrm{~cm} \mathrm{~s}^{-1}$.

\subsection{Linear GJ: influence of the resistance value on the AP velocity}

In the following cases, we changed the value of the resistance for the gap junctions. We used six different resistance values: $R=0.015 \mathrm{k} \Omega, R=0.03 \mathrm{k} \Omega, R=0.06 \mathrm{k} \Omega$,
$R=0.075 \mathrm{k} \Omega, R=0.12 \mathrm{k} \Omega, R=0.15 \mathrm{k} \Omega$. The outcome is that the higher the resistance, the lower the AP velocity. We show in Figure 6 the corresponding graph.

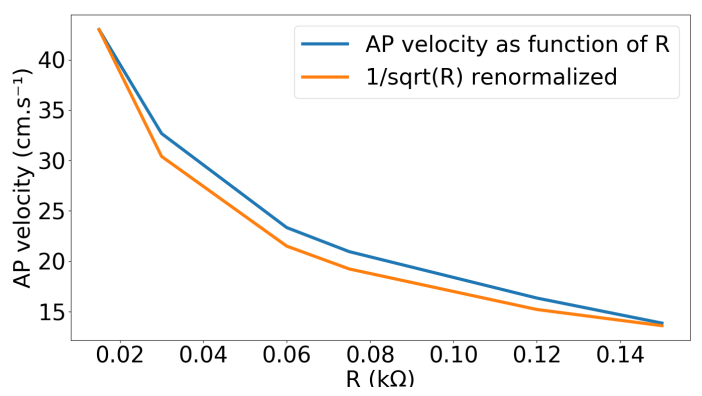

Figure 6. AP velocity as a function of the resistance of the GJCs, on the glued 99-cells problem. We see that the decrease of the velocity is not linear, but as the square root of the resistance.

\subsection{Linear GJ: influence of the GJ density on the AP velocity}

We also studied how the velocity depends on the density of the interfaces along the main cell path, or similarly on the length of the cells. To this aim, we designed a singleline network of a fixed length with cells of varying length (and hence the number of cells, and hence the number of interfaces to cross). We tested this problem with both the linear Gap Junction model and the non-linear one.

We chose to represent five cases: 10 cells of $100 \mu \mathrm{m}$ length, 20 cells of $50 \mu \mathrm{m}$ length, 50 cells of $20 \mu \mathrm{m}$ length, 5 cells of $200 \mu \mathrm{m}$ length and 2 cells of $500 \mu \mathrm{m}$ length. On Figure 7, we present the AP velocity as a function of the cell length. From this figure, we infer that the AP ve-

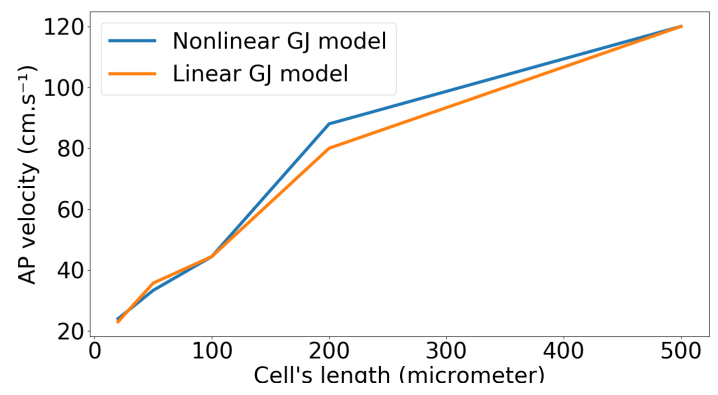

Figure 7. AP velocity as function of the cell length, hence, of the density of gap junction along the main cell path.

locity depends on the density of Gap Junction the AP has to cross. This confirms the impact of the Gap Junctions on the propagation at the microscopic scale. To extract a 
more reliable velocity for cells with great length $(200 \mu \mathrm{m}$ or above), further testing with a longer network should be completed.

\subsection{Example of non-linear GJ model}

We implemented a non-linear GJ model from the results of [5], that got derived from experimentations on rat heart cells. With the same parameters as the Linear GJ simulation, but replacing the linear GJ model by the previously mentioned model, we run a simulation. Figure 8 shows the transmembrane voltages measured for this case. This

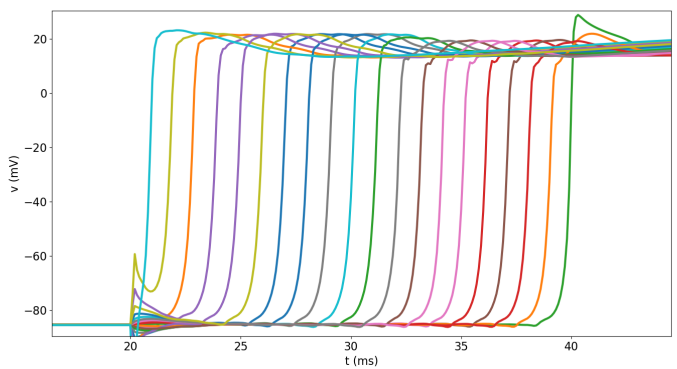

Figure 8. Voltages measured on the blue dots shown in Figure 3 for the nonlinear GJ problem. The action potential takes $19 \mathrm{~ms}$ to cross $0.817 \mathrm{~cm}$, which gives a velocity of $43 \mathrm{~cm} \mathrm{~s}^{-1}$.

result is not distinguishable from the linear problem with $R=0.015 \mathrm{k} \Omega$.

\subsection{Linear GJ: example with too high re- sistance on the membrane between two cells}

We implemented a case where the resistance between two cells (the $38^{\text {th }}$ and the $39^{\text {th }}$ ) is set to $1.5 \mathrm{k} \Omega$. Figure 9 shows the transmembrane voltages measured on the blue dots in Figure 3, which shows the expected block of propagation between cells 38 and 39 .

\section{Conclusion}

From these different results we were able to show the complex role of individual gap junctions on the overall averaged velocity of an action potential. The influence of the different models is to be explored further, with varying parameters, and methods, to confirm the results above. In particular, comparing simulation results with the experimental output from ref [5] could provide a decent way to assess wether our model provides a realistic propagation.

Yet, it remains many questions regarding the influence of gap junctions on the electrical activities of the heart tis-

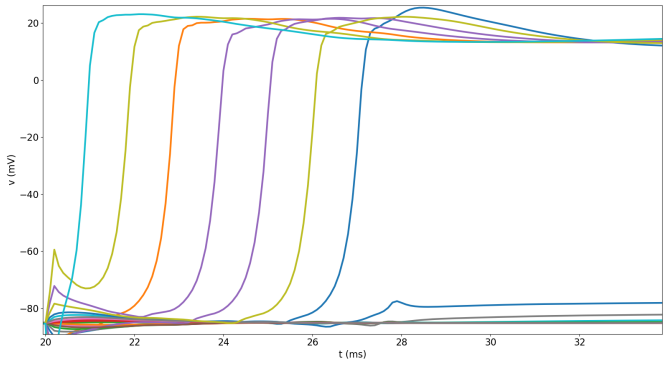

Figure 9. Voltages measured on the blue dots shown in figure 3 with $R=1.5 \mathrm{k} \Omega$ between the $38^{\text {th }}$ and the $39^{\text {th }}$ cells. We can see that the $39^{\text {th }}$ cell failed to depolarize and comes back to rest.

sues, in particular when alterations occur. As homogenized model don't allow such studies, our model seems to provide a reasonable point to start.

\section{References}

[1] Bécue PE, Coudière Y, Potse M. A Three-Dimensional Computational Model of Action Potential Propagation Through a Network of Individual Cells. In Computing in Cardiology Conference (CinC). 2017; 1081-1084.

[2] de Bakker JM, van Capelle FJ, Janse MJ, Tasseron S, Vermeulen JT, de Jonge N, Lahpor JR. Slow Conduction in the Infarcted Human Heart. 'Zigzag' Course of Activation. Circulation 1993;88(3):915-926. ISSN 0009-7322.

[3] Colli-Franzone P, Savaré G. Degenerate Evolution Systems Modeling the Cardiac Electric Field at Micro- and Macroscopic Level. Basel: Birkhäuser Basel. ISBN 978-3-03488221-7, 2002; 49-78.

[4] Bécue PE, Caro F, Potse M, Coudière Y. Theoretical and Numerical Study of Cardiac Electrophysiology Problems at the Microscopic Scale. SIAM Conference on the Life Sciences (LS16), July 2016. URL https://hal.inria.fr/hal-01405837. Poster.

[5] Davidović A, Coudière Y, Desplantez T, Poignard C. Microscopic Modelling of the Non-Linear Gap Junction Channels. In 2015 Computing in Cardiology Conference (CinC). Nice, France, September 2015; URL https://hal.inria.fr/hal-01418702.

[6] Bochev P, Lehoucq RB. On the Finite Element Solution of the Pure Neumann Problem. SIAM Review 2005;47(1):50 66.

Address for correspondence:

Pierre-Elliott Bécue

Maison de la Simulation USR 3441, Bâtiment 565 - Digiteo, CEA Saclay, 91191 Gif-sur-Yvette cédex, France pierre-elliott.becue@inria.fr 